\title{
Automatic Proofs of Protocols via Program Transformation
}

\author{
Fabio Fioravanti ${ }^{1}$, Alberto Pettorossi ${ }^{2}$, Maurizio Proietti ${ }^{3}$ \\ (1) Computer Science Department, University of L'Aquila, I-67100 L'Aquila, Italy \\ fioravanti@di.univaq.it \\ (2) DISP, University of Roma Tor Vergata, I-00133 Roma, Italy \\ pettorossi@info.uniroma2.it \\ (3) IASI-CNR, Viale Manzoni 30, I-00185 Roma, Italy \\ proietti@iasi.rm.cnr.it
}

\begin{abstract}
We propose a method for the specification and the automated verification of temporal properties of protocols which regulate the activities of multiagent systems. The set of states of those systems may be infinite so that, in general, the verification of a property of a multiagent system cannot be performed by an exhaustive inspection. We specify a given multiagent system by means of a constraint logic program $P$ with locally stratified negation, and we specify a given temporal property to be verified by means of an atomic formula $A$. In order to verify that the given temporal property holds, we transform the program $P$ into an equivalent program $T$ such that the fact $A \leftarrow$ belongs to $T$. Our transformation method consists of a set of rules and an automatic strategy that guides the application of the rules. Our method is sound for verifying properties of protocols that are expressible in the CTL logic [5]. Although our method is incomplete for proving properties of infinite state systems, it is able to verify important properties of several protocols which are used in practice.
\end{abstract}

\subsection{Introduction}

Many models of computation have been considered in the literature. Among these are: (i) the centralized, sequential model based on the von Neumann architecture, (ii) the centralized, parallel model based on the parallel random machine with the 'concurrent-read and exclusive-write' restriction on its registers, and (iii) the distributed, multiagent system model. Other models of computation proposed in the literature include those based on rewriting systems, logical deductions, quantum computations, and DNA computations. In this paper we want to consider, in particular, the multiagent system model and we want to analyze a few issues that we think are of major relevance for that model.

The multiagent system model can be understood as a set of agents, each of which can be viewed as a von Neumann computer. These agents inter- 
act with each other and cooperate by exchanging messages with the aim of achieving a common goal, maybe in the presence of antagonistic agents. The cooperation among agents is realized via protocols that ensure suitable global properties of the multiagent system. While computation progresses, a global knowledge of the system of agents is built up, starting from a local, maybe imprecise, knowledge of each individual agent. In the multiagent model the following concepts are important: (i) the local computations (also called local rules), which regulate the activity of each agent, (ii) the protocols (also called metarules), which establish the way agents interact with each other, (iii) the communications, which specify the messages that can be exchanged among agents, and (iv) the distributivity property, which indicates the degree of interaction among agents.

Before illustrating these concepts, let us present a simple example of multiagent system that will help the reader to fix his/her own ideas. In this example we consider the problem of computing a directed spanning tree of a given undirected, connected, finite graph $G=\langle N, E\rangle$, where $N$ is a set of nodes and $E$ is a set of edges (that is, ordered pairs of nodes). Since $G$ is undirected we assume that for every edge $\langle n, m\rangle$ there exists the symmetric edge $\langle m, n\rangle$. For reasons of simplicity, we also assume that in $G$ there is no edge of the form $\langle n, n\rangle$. This spanning tree is computed by several cooperating agents, each of which acting at a node of the given graph $G$. For every node $n \in N$ we define the two sets $P(n)=\{p \mid\langle p, n\rangle \in E\}$ and $S(n)=\{s \mid\langle n, s\rangle \in E\}$, which are the sets of the so called predecessor nodes of $n$ and successor nodes of $n$, respectively.

The agent at node $n$ can communicate only with the agents which are at the nodes of $P(n) \cup S(n)$, and the messages it sends are based only on the local knowledge it has. At the end of the computation, when the spanning tree has been computed, each agent knows the edges which insist on its node and belong to the computed spanning tree.

The multiagent system has to construct a directed spanning tree of the given graph $G$ with a given node $n_{0}$ as its root. During the execution of the algorithm a node may be either unmarked or marked. Initially all nodes are unmarked. The computation performed by the agents at the nodes is done according to the following local rules $R 1-R 3$, which transform the given graph $G$ into a directed spanning tree of $G$ with root $n_{0}$.

(i) Rule $R 1$ is applied by the agent at the root node $n_{0}$. This agent deletes all edges arriving at the root, marks the root, and sends a mark-token to every node in $S\left(n_{0}\right)$.

(ii) Rule $R 2$ is applied by an agent at an unmarked, non-root node $n$ iff there is at least one incoming mark-token from a node, say $i$, of $P(n)$. The agent at node $n$ deletes all edges arriving at $n$ from a node different from $i$, marks the node $n$, and sends a mark-token to every node of $S(n)$.

(iii) Rule $R 3$ is applied by the agent at a marked node $n$ iff all agents at the nodes in $S(n)$ have sent an end-token to node $n$. In particular, rule $R 3$ is 
applied at every node $n$ such that $S(n)=\emptyset$. The agent at node $n$ applies rule $R 3$ by sending an end-token to each node in $P(n)$. (Notice that each time this rule is applied, $P(n)$ has exactly one element.) This rule $R 3$ is needed for detecting the termination of the algorithm.

The algorithm terminates iff every node in $S\left(n_{0}\right)$ has sent an end-token to the root $n_{0}$. One can show that when this happens, the given graph $G$ has been transformed into a directed subgraph $T$ of the original graph $G$ and $T$ is a tree with root $n_{0}$.

In this spanning tree example the protocol consists of the metarules with which every agent should comply. The protocol establishes that: (i) all nodes are initially unmarked and the fixed node $n_{0}$ is the root of the spanning tree to be computed, (ii) the local rules are applied in an atomic way, in the sense that when one of them is applied to a node $n$, no rule can be applied to a node of $P(n) \cup S(n)$, and (iii) the termination of the algorithm occurs when the root $n_{0}$ has received an end-token from each of its children.

Communications among agents take place by sending messages. In the case of our spanning tree algorithm, these messages are either mark-tokens or end-tokens. In general, the messages may be any first-order or higher-order value. They may also be agents themselves [3]. In this case the agents sent as messages, once they reach destination, will operate in a concurrent way together with the agents residing at the destination nodes. In a multiagent system messages may also modify the topology of the communication channels while the computation proceeds. This phenomenon can be modeled within, for instance, the $\pi$-calculus [16].

The notion of distributivity in a multiagent system is related to the amount of global knowledge which is shared by the individual agents during the computation. We say that distributivity is high if every agent in the multiagent system knows a small amount of global knowledge, and it is low if this amount is big. We will not give a formal definition of distributivity: for our purposes here it will be enough to say that the spanning tree algorithm that we have described above, has high distributivity, while the usual, centralized algorithm which performs a depth-first visit of the given graph and keeps a global representation of it, has low distributivity.

The main objective of this paper is to present a technique for proving correctness of protocols. Before entering into this matter, we would like to stress the relevance of the correctness of protocols for multiagent systems by considering the familiar example of the $n$-queens problem for $n=4$. We assume that each queen is a distinct agent. A position of a queen in the $4 \times 4$ board is denoted by a pair of numbers in $\{1,2,3,4\} \times\{1,2,3,4\}$. For instance, a queen in position $\langle 1,2\rangle$ is placed in the first row and in the second column.

Let us consider the initial configuration whose positions are: $\langle 1,1\rangle,\langle 2,2\rangle$, $\langle 3,3\rangle$, and $\langle 4,4\rangle$ (that is, the queens are in the diagonal of the board from bottom-left to top-right). Let us also assume the metarules (or protocol) by which: (i) one queen at a time may make a move, while all other queens do not 
move, and (ii) a queen may go only to a position which is safe. Under these metarules there is no solution to the 4-queens problem because no queen can move.

However, there is a solution to the 4-queens problem if we consider the following metarules: (i) each queen can move only along her column, and (ii) any two mutually attacking queens make their next moves, while the other queens do not move, so that after the move, at least one of the two queens is free from attacks by any other queen. (We assume that each queen knows whether or not she is free from attacks by any other queen, and in this sense she has a global knowledge of the board.)

This protocol is correct, in the sense that there exists a sequence of board configurations which leads to a final configuration where no queen is under attack. One such a sequence is the following:

$$
\begin{aligned}
& \langle 1,1\rangle,\langle 2,2\rangle,\langle 3,3\rangle,\langle 4,4\rangle \rightarrow \quad \text { \{moves of queens } 1 \text { and } 2\} \\
\rightarrow & \langle 1,2\rangle,\langle 2,4\rangle,\langle 3,3\rangle,\langle 4,4\rangle \rightarrow \\
\rightarrow & \langle 1,2\rangle,\langle 2,4\rangle,\langle 3,1\rangle,\langle 4,3\rangle .
\end{aligned}
$$

Notice that the protocol we have presented, leaves unspecified the order in which the pairs of mutually attacking queens should be considered.

\subsection{Specifying Protocols Using Logic Programs}

In this section we present a method for specifying protocols and their properties by means of logic programs.

The behaviour of a multiagent system that evolves over time according to a given protocol, can be modeled by means of a state transition system. This modeling approach has been used in model checking techniques for the formal verification of concurrent systems [5]. A state is a configuration of a system which is identified by an assignment of values to the system variables. A transition between states models an action performed by an agent.

Formally, a state transition system is given by: (i) a set $S$ of states, (ii) an initial state $s_{0} \in S$, and (iii) a transition relation $t \subseteq S \times S$. We assume that $t$ is a total relation, that is, for every state $s_{1} \in \bar{S}$ there exists a state $s_{2} \in S$, called successor state of $s_{1}$, such that $t\left(s_{1}, s_{2}\right)$ holds. A computation path starting from a state $s_{1}$ is an infinite sequence of states $s_{0} s_{1} \ldots$ such that, for every $i \geq 0, t\left(s_{i}, s_{i+1}\right)$ holds. In this section and in the next one, we deal with finite state systems, that is, we assume that the system variables range over finite sets of values and, thus, the set of states is finite.

The transition relation $t$ can be defined by clauses of a logic program. For instance, the finite state system depicted in Figure 1.1 can be specified by the relation $t$ defined by the following four unit clauses:

$$
t\left(s_{0}, s_{1}\right) \leftarrow \quad t\left(s_{1}, s_{0}\right) \leftarrow \quad t\left(s_{1}, s_{2}\right) \leftarrow \quad t\left(s_{2}, s_{2}\right) \leftarrow
$$

The properties of the evolution over time of a state transition system are specified by using a temporal logic called Computation Tree Logic (or CTL, for short [5]). We suppose that, for each state $s \in S$, we are given a set of 


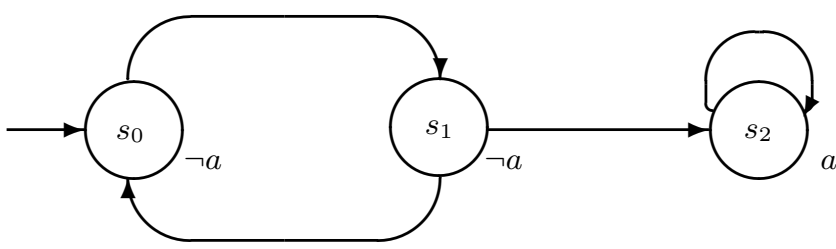

Figure 1.1. A finite state transition system. The formula $\varphi$ placed near state $s_{i}$ indicates that $\varphi$ holds in $s_{i} . s_{0}$ is the initial state.

elementary properties that hold in $s$. These elementary properties are specified by a binary relation elem such that elem $(s, p)$ holds iff the elementary property $p$ holds in $s$. The relation elem can be defined by a logic program. For instance, if we assume that no elementary property holds in $s_{0}$ and $s_{1}$, and the elementary property $a$ holds in $s_{2}$ (see Figure 1.1), then the relation elem is defined by the following unit clause:

$$
\operatorname{elem}\left(s_{2}, a\right) \leftarrow
$$

The formulas of CTL are built from the given set of elementary properties by using: (i) the connectives: $\neg$ ('not') and $\wedge$ ('and'), (ii) the following quantifiers along a computation path: $g$ ('for all states on the path' or 'globally'), $f$ ('there exists a state in the path' or 'in the future'), $x$ ('next time'), and $u$ ('until'), and (iii) the quantifiers over computation paths: $a$ ('for all paths') and $e$ ('there exists a path'). In this paper we will consider only the subset of the formulas of CTL that can be constructed by using the connectives and the two combinations ef and af of quantifiers. Thus, we assume that the syntax of a temporal formula $\varphi$ is as follows:

$$
\varphi::=p|\neg \varphi| \varphi_{1} \wedge \varphi_{2} \mid \text { ef } \varphi \mid \text { af } \varphi
$$

where $p$ is an elementary property. We also use the following abbreviations: (i) $e g \varphi \equiv \neg a f \neg \varphi$ and (ii) $a g \varphi \equiv \neg e f \neg \varphi$.

The semantics of temporal formulas is given by: (i) a Kripke structure $\mathcal{K}[5]$, which is a state transition system together with a relation elem specifying a set of elementary properties for each state $s$ of $\mathcal{K}$, and (ii) a satisfaction relation $\mathcal{K}, s=\varphi$ denoting that a formula $\varphi$ holds in a state $s$ of $\mathcal{K}$. The relation $\mathcal{K}, s=\varphi$ is inductively defined as follows:

$\mathcal{K}, s=p$ iff $p$ is an elementary property such that elem $(s, p)$ holds

$\mathcal{K}, s=\neg \varphi$ iff it is not the case that $\mathcal{K}, s \models \varphi$

$\mathcal{K}, s=\varphi_{1} \wedge \varphi_{2}$ iff $\mathcal{K}, s=\varphi_{1}$ and $\mathcal{K}, s=\varphi_{2}$

$\mathcal{K}, s=$ ef $\varphi$ iff there exists a computation path $s_{0} s_{1} \ldots$ such that (i) $s=s_{0}$ and (ii) for some $n \geq 0$ we have that $\mathcal{K}, s_{n} \models \varphi$

$\mathcal{K}, s=$ af $\varphi$ iff for all computation paths $s_{0} s_{1} \ldots$ if $s=s_{0}$ then there exists $n \geq 0$ such that $\mathcal{K}, s_{n} \models \varphi$.

For instance, let $\mathcal{K}_{0}$ be the Kripke structure consisting of the transition system of Figure 1.1, where the elementary property $a$ holds in state $s_{2}$ only. Then the following properties hold: 


$$
\begin{array}{llrl}
\mathcal{K}_{0}, s_{0} & =\text { ef } a & \mathcal{K}_{0}, s_{0} \models \text { af ef } a \\
\mathcal{K}_{0}, s_{0}=\neg \text { af } a & \mathcal{K}_{0}, s_{0} \models \text { ag ef } a
\end{array}
$$

In order to verify that a temporal formula $\varphi$ holds in the initial state $s_{0}$ of a given Kripke structure $\mathcal{K}$, we encode the satisfaction relation $\mathcal{K}, s_{0}=\varphi$ as a predicate $\operatorname{sat}\left(s_{0}, \varphi\right)$ defined by a logic program $P_{\mathcal{K}}$. This program can be constructed by induction on the structure of the formula $\varphi$ as follows [9]:

S1. $\operatorname{sat}(X, F) \leftarrow \operatorname{elem}(X, F)$

S2. $\operatorname{sat}(X, \neg F) \leftarrow \neg \operatorname{sat}(X, F)$

S3. $\operatorname{sat}(X, F 1 \wedge F 2) \leftarrow \operatorname{sat}(X, F 1) \wedge \operatorname{sat}(X, F 2)$

S4. $\operatorname{sat}(X$, ef $F) \leftarrow \operatorname{sat}(X, F)$

S5. $\operatorname{sat}(X$, ef $F) \leftarrow t(X, Y) \wedge \operatorname{sat}(Y$, ef $F)$

S6. $\operatorname{sat}(X$, af $F) \leftarrow \operatorname{sat}(X, F)$

together with, for each state $s \in S$, a clause of the form:

S7. sat $(s$, af $F) \leftarrow \operatorname{sat}\left(s_{1}\right.$, af $\left.F\right) \wedge \ldots \wedge \operatorname{sat}\left(s_{k}\right.$, af $\left.F\right)$

where $s_{1}, \ldots, s_{k}$ are the successor states of $s$. Program $P_{\mathcal{K}}$ also includes the clauses that define the relations elem and $t$ for the given Kripke structure $\mathcal{K}$.

In program $P_{\mathcal{K}}$ clauses S4 and S5 express that the formula ef $\varphi$ holds in a state $s$ iff either $\varphi$ holds in $s$ or ef $\varphi$ holds in a successor state of $s$. Clauses S6 and S7 express that the formula af $\varphi$ holds in a state $s$ iff either $\varphi$ holds in $s$ or af $\varphi$ holds in all successor states of $s$.

Program $P_{\mathcal{K}}$ is a logic program with locally stratified negation and, thus, it has a unique perfect model, denoted by $M\left(P_{\mathcal{K}}\right)$ [1]. For every Kripke structure $\mathcal{K}$, state $s_{0}$, and temporal formula $\varphi$, we have that [9]:

$$
\mathcal{K}, s_{0} \models \varphi \quad \text { iff } \quad \operatorname{sat}\left(s_{0}, \varphi\right) \in M\left(P_{\mathcal{K}}\right)
$$

Unfortunately, it is often the case that we cannot use SLDNF resolution $[1,13]$ to check whether or not $\operatorname{sat}\left(s_{0}, \varphi\right)$ belongs to $M\left(P_{\mathcal{K}}\right)$, because SLDNF resolution may not terminate for the goal $\operatorname{sat}\left(s_{0}, \varphi\right)$ for many temporal formulas $\varphi$. This is due to the presence of clauses S5 and S7 and to the fact that SLDNF resolution is not able to detect the presence of infinite loops.

Tabled resolution [4] overcomes this limitation of SLDNF resolution in the case of finite state systems by maintaining a table of predicate calls and avoiding their repeated evaluation. However, tabled resolution is no longer effective when we consider logic programs encoding infinite state systems, that is, when the transition relation $t$ is infinite (see Section 1.4). Indeed, in that case, when we evaluate the goal $\operatorname{sat}\left(s_{0}, \varphi\right)$ by using tabled resolution, infinitely many different predicate calls may be generated.

In the next section we will show a technique based on program transformation [2] for checking whether or not $\operatorname{sat}\left(s_{0}, \varphi\right)$ belongs to $M\left(P_{\mathcal{K}}\right)$. This technique is complete for programs $P_{\mathcal{K}}$ encoding finite state systems. Moreover, in Section 1.4 we will show that our technique is also able to verify that sat $\left(s_{0}, \varphi\right)$ belongs to $M\left(P_{\mathcal{K}}\right)$ also for a large class of programs $P_{\mathcal{K}}$ encoding infinite state systems. 


\subsection{Transformation Rules and Strategies for the Verification of Protocols}

In this section we describe our method for verifying temporal properties of protocols. This method is based on suitable transformation rules (see rules R1-R4 below) and on a transformation strategy, called UFV (short for Unfold/Fold Verification), for guiding the application of these rules. Our verification method consists of two steps as indicated below.

\section{The Verification Method.}

Step 1. Given a Kripke structure $\mathcal{K}$, an initial state $s_{0}$, and a temporal formula $\varphi$, we construct a program $P_{\mathcal{K}}$ such that $\mathcal{K}, s_{0} \models \varphi$ iff $\operatorname{sat}\left(s_{0}, \varphi\right) \in M\left(P_{\mathcal{K}}\right)$ as indicated in Section 1.2.

Step 2. We introduce the clause $\delta_{0}:$ new $0 \leftarrow \operatorname{sat}\left(s_{0}, \varphi\right)$, where new0 is a new predicate symbol. Then we apply the transformation rules R1-R4 according to the transformation strategy $U F V$ and from program $P_{\mathcal{K}} \cup\left\{\delta_{0}\right\}$ we derive a transformed program $T$ such that: new $0 \in M\left(P_{\mathcal{K}} \cup\left\{\delta_{0}\right\}\right)$ iff new $0 \in M(T)$. Finally, we inspect program $T$ and

(i) if the unit clause new $0 \leftarrow$ occurs in $T$ then $\mathcal{K}, s_{0}=\varphi$, and

(ii) if no clause with head new0 occurs in $T$ then $\mathcal{K}, s_{0} \not \models \varphi$.

The correctness of our verification method is a consequence of the following facts: (i) by construction we have that $\mathcal{K}, s_{0}=\varphi$ iff $\operatorname{sat}\left(s_{0}, \varphi\right) \in M\left(P_{\mathcal{K}}\right)$, (ii) since $\delta_{0}:$ new $0 \leftarrow \operatorname{sat}\left(s_{0}, \varphi\right)$ is the only clause that defines new0 in $P_{\mathcal{K}} \cup$ $\left\{\delta_{0}\right\}$, we have that $\operatorname{sat}\left(s_{0}, \varphi\right) \in M\left(P_{\mathcal{K}}\right)$ iff new $0 \in M\left(P_{\mathcal{K}} \cup\left\{\delta_{0}\right\}\right)$, (iii) since the transformation rules, when applied according to the transformation strategy $U F V$, preserve the perfect model, new $0 \in M\left(P_{\mathcal{K}} \cup\left\{\delta_{0}\right\}\right)$ iff new0 $\in M(T)$, and finally, (iv) by the definition of perfect model, (iv.1) if new0 $\leftarrow$ occurs in $T$ then new $0 \in M(T)$ and (iv.2) if no clause with head new0 occurs in $T$ then new0 $\notin M(T)$.

Let us return to our example of Figure 1.1 and let us suppose that we want to verify that $\mathcal{K}_{0}, s_{0} \models \neg$ ef $\neg$ ef $a$ holds, that is, for every state reachable from the initial state $s_{0}$ it is possible to get to a state where $a$ holds. Let $P_{0}$ be the program constructed at Step 1 as indicated in Section 1.3. Step 2 of our verification method consists in transforming the program $P_{0} \cup\left\{\delta_{0}\right\}$ where $\delta_{0}$ is the clause new $0 \leftarrow \operatorname{sat}\left(s_{0}, \neg\right.$ ef $\neg$ ef $\left.a\right)$ into a program where new $0 \leftarrow$ occurs. We will present this transformation in Section 1.3.3.

\subsubsection{The Transformation Rules}

The process of transforming a given program $P_{\mathcal{K}}$ thereby deriving program $T$, can be formalized as a sequence $P_{0}, \ldots, P_{n}$ of programs, called a transformation sequence, where: (i) $P_{0}=P_{\mathcal{K}}$, (ii) $P_{n}=T$, and (iii) for $i=0, \ldots, n-1$, program $P_{i+1}$ is obtained from program $P_{i}$ by applying one of the transformation rules listed below. These rules are variants of the rules considered in the literature for transforming logic programs (see, in particular, [20, 21]). 
The atomic definition rule allows us to introduce a new predicate definition by adding to program $P_{i}$ a new clause whose body consists of one atom only. We can use this rule to add clause $\delta_{0}$ to program $P_{\mathcal{K}}$ at the beginning of Step 2 of our verification method.

R1. Atomic Definition. We introduce a new clause, called a definition, of the form:

$$
\delta: \operatorname{newp}\left(X_{1}, \ldots, X_{m}\right) \leftarrow A
$$

where: (i) newp is a predicate symbol not occurring in $P_{0}, \ldots, P_{i}$, (ii) $X_{1}, \ldots$, $X_{m}$ are the variables occurring in $A$, and (iii) the predicate of $A$ occurs in $P_{0}$. By atomic definition (or definition, for short), we derive the new program $P_{i+1}=P_{i} \cup\{\delta\}$. For $i \geq 0$, Defs $_{i}$ denotes the set of definitions introduced during the transformation sequence $P_{0}, \ldots, P_{i}$. In particular, Defs $s_{0}=\emptyset$.

The unfolding rule corresponds to a symbolic computation step. It replaces a clause $\gamma$ in $P_{i}$ by the set of all clauses that can be derived by applying a resolution step w.r.t. a literal $L$ occurring in the body of $\gamma$. We have a positive and a negative unfolding rule, according to the case where $L$ is a positive or negative literal. Notice that in the negative unfolding rule (see case R2n below) the literal $L$ should be either valid or failed. We say that an atom $A$ is valid in a program $P$ iff there exists a unit clause $H \leftarrow$ in $P$ such that $A$ is an instance of $H$. We say that an atom $A$ is failed in $P$ iff there exists no clause $H \leftarrow G$ in $P$ such that $A$ is unifiable with $B$. The negated atom $\neg A$ is valid iff $A$ is failed and $\neg A$ is failed iff $A$ is valid.

R2. Unfolding. Let $\gamma: H \leftarrow G_{1} \wedge L \wedge G_{2}$ be a clause in $P_{i}$ and let $P_{i}^{\prime}$ be a variant of $P_{i}$ without common variables with $\gamma$. We consider the following two cases.

(R2p: Positive Unfolding) Let $L$ be a positive literal. By unfolding $\gamma$ w.r.t. $L$ we derive the set of clauses

$\Gamma:\left\{\left(H \leftarrow G_{1} \wedge G \wedge G_{2}\right) \vartheta \mid\right.$ (i) $K \leftarrow G$ is a clause in $P_{i}^{\prime}$ and

(ii) $L$ and $K$ are unifiable with mgu $\vartheta\}$

We derive the new program $P_{i+1}=\left(P_{i}-\{\gamma\}\right) \cup \Gamma$.

(R2n: Negative Unfolding) Let $L$ be a negative literal.

(i) If $L$ is valid in $P_{i}^{\prime}$, then by unfolding $\gamma$ w.r.t. $L$ we derive the clause $\eta: H \leftarrow G_{1} \wedge G_{2}$

and we derive the new program $P_{i+1}=\left(P_{i}-\{\gamma\}\right) \cup\{\eta\}$.

(ii) If $L$ is failed in $P_{i}^{\prime}$, then by unfolding $\gamma$ w.r.t. $L$ we derive the new program $P_{i+1}=P_{i}-\{\gamma\}$.

The atomic folding rule allows us to replace an atom $A$ which is an instance of the body of a definition by the corresponding instance of the head of the definition.

R3. Atomic Folding. Let $\gamma: H \leftarrow G_{1} \wedge L \wedge G_{2}$ be a clause in $P_{i}$ and let $\delta: N \leftarrow A$ be a clause in $\operatorname{Defs}_{i}$ without common variables with $\gamma$. We consider the following two cases. 
(R3p: Positive Folding) Let $L$ be the atom $A \vartheta$ for some substitution $\vartheta$. By folding $\gamma$ w.r.t. A using $\delta$ we derive the clause

$\eta: H \leftarrow G_{1} \wedge N \vartheta \wedge G_{2}$

and we derive the new program $P_{i+1}=\left(P_{i}-\{\gamma\}\right) \cup\{\eta\}$.

(R3n: Negative Folding) Let $L$ be the negated atom $\neg A \vartheta$. By folding $\gamma$ w.r.t. $\neg A$ using $\delta$, we derive the clause

$\eta: H \leftarrow G_{1} \wedge \neg N \vartheta \wedge G_{2}$

and we derive the new program $P_{i+1}=\left(P_{i}-\{\gamma\}\right) \cup\{\eta\}$.

The following clause removal rule may be used for removing from $P_{i}$ a redundant clause $\gamma$, that is, a clause $\gamma$ such that $M\left(P_{i}\right)=M\left(P_{i}-\{\gamma\}\right)$. Let us first introduce the following definitions. The set of useless predicates in a program $P$ is the maximal set $U$ of predicate symbols occurring in $P$ such that a predicate $p$ is in $U$ iff for every clause $p(\ldots) \leftarrow G$ in $P$, the body $G$ is of the form $G_{1} \wedge q(\ldots) \wedge G_{2}$ for some predicate $q$ in $U$. A clause is useless iff the predicate in its head is useless.

R4. Clause Removal. Let $\gamma$ be a clause in $P_{i}$. By clause removal we derive the new program $P_{i+1}=P_{i}-\{\gamma\}$ if one of the following cases occurs:

(R4s: Removal of Subsumed Clause) $\gamma$ is a clause $H \leftarrow G$ and $H$ is valid in $P_{i}-\{\gamma\}$

(R4u: Removal of Useless Clause) $\gamma$ is useless in $P_{i}$.

\subsubsection{The Transformation Strategy}

During Step 2 of our verification method the transformation rules are applied according to the following transformation strategy $U F V$.

\section{The Transformation Strategy UFV.}

Input: A program $P_{\mathcal{K}}$ and a clause $\delta_{0}:$ new $0 \leftarrow \operatorname{sat}\left(s_{0}, \varphi\right)$. They encode the Kripke structure $\mathcal{K}$ and the property $\varphi$ to be verified in the initial state $s_{0}$. Output: A transformed program $T$ such that new $0 \in M\left(P_{\mathcal{K}} \cup\left\{\delta_{0}\right\}\right)$ iff new0 $\in$ $M(T)$.

Phase A. $P_{A}:=\emptyset ; \quad$ Defs $:=\left\{\delta_{0}\right\} ; \quad \Delta:=\left\{\delta_{0}\right\} ;$

WHILE there exists a clause $\delta \in \Delta$

DO $\operatorname{Unfold}(\delta, \Gamma)$

Define\&Fold $(\Gamma$, Defs, NewDefs,$\Phi)$;

$P_{A}:=P_{A} \cup \Phi ; \quad$ Defs $:=$ Defs $\cup$ NewDefs $; \quad \Delta:=(\Delta-\{\delta\}) \cup$ NewDefs END-WHILE

Phase B. Remove\& Unfold $\left(P_{A}, T\right)$

The UFV strategy is divided into two phases: Phase $\mathrm{A}$ and Phase $\mathrm{B}$. Phase A takes program $P_{\mathcal{K}}$ and clause $\delta_{0}$ as input and returns program $P_{A}$ as output. Initially program $P_{A}$ is the empty set of clauses. During Phase A we use the following two sets of clauses: (1) Defs, which is the set of definitions 
introduced during the transformation process, and (2) $\Delta$, which is the set of definitions that have been introduced but not yet unfolded. At the beginning of Phase A we apply the definition rule and we add clause $\delta_{0}$ to Defs and $\Delta$. Then the strategy performs a WHILE-DO loop whose body consists of applications of the unfolding rule according to the Unfold procedure (see below), followed by applications of the definition and folding rules according to the Define\& Fold procedure (see below).

The Unfold procedure takes a definition clause $\delta \in \Delta$ and derives a set $\Gamma$ of clauses by applying one or more times the positive unfolding rule starting from clause $\delta$. Every clause in $\Gamma$ is of the form $H \leftarrow L_{1} \wedge \ldots \wedge L_{n}$, where $n \geq 0$ and each literal $L_{i}$ is either an atom of the form $\operatorname{sat}(s, \psi)$ or a negated atom of the form $\neg \operatorname{sat}(s, \psi)$. The Define\& Fold procedure takes as input: (i) the set $\Gamma$ of clauses and (ii) the set Defs of definitions, and returns as output: (i) a set NewDefs of new definitions and (ii) a set $\Phi$ of transformed clauses. NewDefs is the set of definitions of the form newp $\leftarrow$ sat $(s, \psi)$ such that (i.1) a literal of the form either sat $(s, \psi)$ or $\neg$ sat $(s, \psi)$ occurs in the body of a clause in $\Gamma$, and (i.2) there is no definition of the form newq $\leftarrow$ sat $(s, \psi)$ in Defs. The set $\Phi$ consists of: (ii.1) all unit clauses belonging to $\Gamma$, together with (ii.2) all clauses derived by (positive or negative) folding of each clause in $\Gamma$ w.r.t. each sat literal in its body using a definition in Defs $\cup$ NewDefs. After the execution of the Define\&Fold procedure the clauses of $\Phi$ are added to $P_{A}$, the definitions of NewDefs are added to Defs and, in the set $\Delta$, clause $\delta$ is replaced by the clauses of NewDefs.

Phase B is realized by the Remove\& Unfold procedure, which transforms $P_{A}$ by repeatedly removing useless clauses, subsumed clauses, and applying the positive and the negative unfolding rule w.r.t. valid or failed literals. Upon termination this procedure returns a program $T$ where new 0 is either valid or failed, that is, either (i) the unit clause new $0 \leftarrow$ occurs in $T$ or (ii) no clause with head new 0 occurs in $T$. In case (i) we have that $\mathcal{K}, s_{0} \models \varphi$ and in case (ii) we have that $\mathcal{K}, s_{0} \not \models \varphi$.

For a Kripke structure $\mathcal{K}$ with a finite set of states the $U F V$ strategy terminates. In particular, only a finite number of definitions will be introduced during the execution of the WHILE-DO loop because only a finite number of distinct atoms of the form $\operatorname{sat}(s, \psi)$ can be generated. Indeed $s$ is an element of a finite set of states and $\psi$ is a (proper or not) subformula of the given formula $\varphi$. Thus, the $U F V$ strategy is a decision procedure for checking whether or not $\mathcal{K}, s_{0}=\varphi$ holds for any given finite state Kripke structure $\mathcal{K}$, initial state $s_{0}$, and temporal formula $\varphi$.

\subsubsection{An Example of Application of the Verification Method}

In this section we will see our transformation strategy in action for the verification of a property of the finite state system of Figure 1.1. We want to show that in that system, for every state which is reachable from the initial state $s_{0}$, it is possible to get to a state where $a$ holds, that is, $\mathcal{K}_{0}, s_{0} \models$ ag ef $a$. The initial program $P_{0}$ which encodes the Kripke structure $\mathcal{K}_{0}$, is the following: 
1. $\operatorname{sat}\left(s_{2}, a\right) \leftarrow$

2. $\operatorname{sat}(S, \neg F) \leftarrow \neg \operatorname{sat}(S, F)$

3. $\operatorname{sat}(S$, ef $F) \leftarrow \operatorname{sat}(S, F)$
4. $\operatorname{sat}\left(s_{0}\right.$, ef $\left.F\right) \leftarrow \operatorname{sat}\left(s_{1}\right.$, ef $\left.F\right)$

5. $\operatorname{sat}\left(s_{1}\right.$, ef $\left.F\right) \leftarrow \operatorname{sat}\left(s_{0}\right.$, ef $\left.F\right)$

6. $\operatorname{sat}\left(s_{1}\right.$, ef $\left.F\right) \leftarrow \operatorname{sat}\left(s_{2}\right.$, ef $\left.F\right)$

7. $\operatorname{sat}\left(s_{2}\right.$, ef $\left.F\right) \leftarrow \operatorname{sat}\left(s_{2}\right.$, ef $\left.F\right)$

Program $P_{0}$ has been obtained by unfolding the program $P_{\mathcal{K}}$ which encodes a generic Kripke structure $\mathcal{K}$, by using the clauses which define the relations elem and $t$ relative to $\mathcal{K}_{0}$ (see Section 1.2). We have not considered the clauses for temporal formulas of the form $F 1 \wedge F 2$ and af $F$ because they are not needed during the application of the transformation strategy.

The clause $\delta_{0}$ is new $0 \leftarrow \operatorname{sat}\left(s_{0}, \neg\right.$ ef $\neg$ ef $\left.a\right)$. (Recall that ag ef $\varphi$ is equivalent to $\neg$ ef $\neg$ ef $\varphi$.) By applying the Unfold procedure, we unfold clause $\delta_{0}$ using clause 2 and we get:

$$
\text { 8. } n e w 0 \leftarrow \neg \operatorname{sat}\left(s_{0}, \text { ef } \neg \text { ef } a\right) \text {. }
$$

Then we apply the Define\&Fold procedure. We introduce the definition:

9. new $1 \leftarrow \operatorname{sat}\left(s_{0}\right.$, ef $\neg$ ef $\left.a\right)$

and we fold clause 8 using clause 9 . We get the following clause:

10. new $0 \leftarrow \neg n e w 1$

At the end of the Define\&Fold procedure, we have that the set $\Phi$ of clauses and the program $P_{A}$ are both equal to $\{$ clause 10$\}$. We also have that Defs $=$ $\left\{\delta_{0}\right.$, clause 9$\}$ and NewDefs $=\Delta=\{$ clause 9$\}$. Thus, we perform again the body of the WHILE-DO loop of Phase A of the UFV strategy. We apply the Unfold procedure and we unfold clause 9. We derive the following clauses:
11. new $1 \leftarrow \neg \operatorname{sat}\left(s_{0}\right.$, ef $\left.a\right)$
13. new $1 \leftarrow \neg \operatorname{sat}\left(s_{2}\right.$, ef $\left.a\right)$
12. new $1 \leftarrow \neg \operatorname{sat}\left(s_{1}\right.$, ef $\left.a\right)$

Thus, $\Gamma=\{$ clause 11 , clause 12 , clause 13$\}$. We apply again the Define\& Fold procedure. The new value of NewDefs is the set of the following three clauses:
14. new $2 \leftarrow \operatorname{sat}\left(s_{0}\right.$, ef $\left.a\right)$
16. new $4 \leftarrow \operatorname{sat}\left(s_{2}\right.$, ef $\left.a\right)$
15. new $3 \leftarrow \operatorname{sat}\left(s_{1}\right.$, ef $\left.a\right)$

which are then used to fold clauses 11,12 , and 13 . These folding steps generate the following three clauses which are added to $P_{A}$ :
17. new $1 \leftarrow \neg$ new 2
19. new $1 \leftarrow \neg$ new 4
18. new $1 \leftarrow \neg$ new 3

Now we apply for each clause in NewDefs the Unfold and Define\&Fold procedures. For instance, starting from clause 14, by positive unfolding we get:

20. new $2 \leftarrow \operatorname{sat}\left(s_{1}\right.$, ef $\left.a\right)$

and then by folding this clause using clause 15 we get:

21. new $2 \leftarrow$ new 3

Analogously, from clauses 15 and 16 by applying the Unfold and Define\& Fold procedures we get:
22. new $3 \leftarrow$ new 2
24. new $4 \leftarrow$
23. new $3 \leftarrow$ new 4
25. new $4 \leftarrow$ new 4 
This concludes Phase A and the derived program $P_{A}$ consists of the clauses $10,17,18,19,21,22,23,24$, and 25 . During Phase B we apply (positive and negative) unfolding and subsumption to the clauses in $P_{A}$ and we get the final program $T$ which consists of the following four unit clauses:
26. new $0 \leftarrow$
28. new $3 \leftarrow$
27. new $2 \longleftarrow$
24. new $4 \longleftarrow$

Thus, since in this program there is the clause new $0 \leftarrow$, we conclude that $\mathcal{K}_{0}, s_{0}=a g$ ef $a$ holds.

\subsection{Verification of Infinite State Systems}

Our transformational approach for the verification of properties of protocols can be extended from finite state systems to infinite state systems. In order to specify infinite state systems we extend the method described in Section 1.2 by using constraint logic programs [15]. These programs generalize logic programs by allowing the bodies of the clauses to contain constraints. Constraints are formulas that define relations over some given domains, such as real numbers, integers, and trees. For our purposes here, constraints are simply first order formulas whose predicate symbols are taken from a distinct set. These constraints can be evaluated by using constraint solvers that are realized by ad hoc algorithms.

The semantics of a constraint $c$ is defined by the usual first order satisfaction relation $\mathcal{D} \models c$, where $\mathcal{D}$ is a fixed interpretation. The notion of perfect model can be extended from logic programs to constraint logic programs in a straightforward way [9].

A transition relation $t$ on an infinite set of states can be specified by using constraints in the body of the clauses that define $t$. For instance, the clause $t(X, Y) \leftarrow X>0 \wedge Y=X+1$ specifies a transition relation on the set of the integer numbers. We will see a more elaborate example in Section 1.4.1.

In order to encode the satisfaction relation $\mathcal{K}, s \models \varphi$ for a Kripke structure $\mathcal{K}$ with an infinite set of states, we can construct a constraint logic program $P_{\mathcal{K}}$ similarly to what we have described in Section 1.2. However, in order to encode the satisfiability of a temporal formula of the form af $\varphi$, the method of Section 1.2 introduces a clause for each state of $\mathcal{K}$ and this is impossible for infinite state systems. Fortunately, this problem can be overcome for a large class of infinite state systems by using constraints as indicated in [9] (see also Section 1.4.1 for an example).

In order to extend our verification method to the case of infinite state systems, we need to extend the transformation rules and the transformation strategy presented in Section 1.3 to the case of constraint logic programs. The extensions of the definition, unfolding, folding, and clause replacement rules can be found in $[8,9]$. Moreover, we will use the following two transformation rules which specifically refer to constraints: (i) Rule R4f, for deleting a clause whose body contains an unsatisfiable constraint, and (ii) Rule R5, for replacing a constraint by an equivalent one. 
R4f. Removal of Clauses with Unsatisfiable Body. Let $\gamma$ be a clause of the form $H \leftarrow c \wedge G$ in $P_{i}$. Suppose that $c$ is unsatisfiable, that is, $\mathcal{D} \models \neg \exists(c)$, where $\exists(c)$ is the existential closure of $c$. Then, we derive the new program $P_{i+1}=P_{i}-\{\gamma\}$.

R5. Constraint Replacement. Let $\gamma_{1}: H \leftarrow c_{1} \wedge G$ be a clause in $P_{i}$. Suppose that for some constraint $c_{2}$, we have that:

$$
\mathcal{D} \models \forall\left(\exists Y_{1} \ldots \exists Y_{k} c_{1} \leftrightarrow \exists Z_{1} \ldots \exists Z_{m} c_{2}\right)
$$

where: (i) $Y_{1}, \ldots, Y_{k}$ are the variables occurring free in $c_{1}$ and not in $\{H, G\}$, (ii) $Z_{1}, \ldots, Z_{m}$ are the variables occurring free in $c_{2}$ and not in $\{H, G\}$, and $\forall(\varphi)$ denotes the universal closure of formula $\varphi$. Then by constraint replacement we derive the clause

$$
\gamma_{2}: H \leftarrow c_{2} \wedge G
$$

and we derive the new program $P_{i+1}=\left(P_{i}-\left\{\gamma_{1}\right\}\right) \cup\left\{\gamma_{2}\right\}$.

The transformation strategy $U F V$ presented in Section 1.3 .2 can be extended to constraint logic programs that encode infinite state systems. During the execution of this strategy, we apply the modified transformation rules for constraint logic programs. In particular, during the execution of the Define\& Fold procedure, when applying the definition rule, we introduce new definitions of the form:

$$
N e w H \leftarrow c(X) \wedge \operatorname{sat}(X, \psi)
$$

where: (i) $X$ is a variable ranging over states, (ii) $c(X)$ is a constraint representing a possibly infinite set of states, and (iii) $\psi$ is a temporal formula.

The main issue that arises when dealing with infinite state systems is that the termination of the $U F V$ strategy is no longer guaranteed. Indeed, for any given temporal formula $\psi$, an infinite number of constrained atoms of the form $c(X) \wedge \operatorname{sat}(X, \psi)$ with non-equivalent constraints may be generated and, thus, an infinite number of non-equivalent definitions may be introduced.

For instance, during the verification of the mutual exclusion property of the Bakery protocol (see Section 1.4.1 below), starting from the initial definition $\delta_{0}:$ new $0 \leftarrow \operatorname{sat}(\langle$ think, 0 , think, 0$\rangle, \neg$ ef unsafe $)$, the $U F V$ strategy introduces an infinite sequence of definitions of the form:

$$
\begin{aligned}
& \delta_{1}: \text { new } 1 \leftarrow A 2=0 \wedge B 2=0 \wedge \operatorname{sat}(\langle\text { think, } A 2 \text {, think, } B 2\rangle \text {, ef unsafe }) \\
& \delta_{2}: \text { new } 2 \leftarrow A 2=1 \wedge B 2=0 \wedge \operatorname{sat}(\langle\text { wait, } A 2 \text {, think, } B 2\rangle \text {, ef unsafe }) \\
& \delta_{3}: \text { new } 3 \leftarrow A 2=3 \wedge B 2=0 \wedge \text { sat }(\langle\text { wait, } A 2 \text {, think, } B 2\rangle \text {, ef unsafe }) \\
& \text {... } \\
& \delta_{k}: \text { newk } \leftarrow A 2=2 k-3 \wedge B 2=0 \wedge \operatorname{sat}(\langle\text { wait }, A 2, \text { think, } B 2\rangle \text {, ef unsafe }) \\
& \text {... }
\end{aligned}
$$

and the $U F V$ strategy does not terminate. We can often overcome this nontermination problem by introducing a generalization operator between clauses, and modifying the Define\&Fold procedure used of the $U F V$ strategy as we now indicate. Suppose that a constrained literal $L$ of the form either $d(X) \wedge$ $\operatorname{sat}(X, \psi)$ or $d(X) \wedge \neg \operatorname{sat}(X, \psi)$ occurs in the body of a clause $\gamma$ belonging 
to the set $\Gamma$ of the clauses derived by the Unfold procedure. In order to fold $\gamma$ w.r.t. $L$ we proceed as follows: (i) if $\gamma$ can be folded using a definition $\delta$ belonging to the set Defs of all definitions introduced so far, then we fold $\gamma$ using $\delta$, otherwise (ii) we consider a clause in Defs of the form:

$$
N e w H 1 \leftarrow c(X) \wedge \operatorname{sat}(X, \psi)
$$

and a clause of the form:

$$
\text { NewH } 2 \leftarrow d(X) \wedge \operatorname{sat}(X, \psi)
$$

and we introduce the generalized clause:

$$
\delta_{\text {gen }}: G e n H \leftarrow \operatorname{genc}(X) \wedge \operatorname{sat}(X, \psi)
$$

where $\mathcal{D} \mid=\forall X(c(X) \rightarrow \operatorname{genc}(X))$ and $\mathcal{D} \models \forall X(d(X) \rightarrow \operatorname{genc}(X))$. Then we fold $\gamma$ using $\delta_{\text {gen }}$. For example, during the verification of the Bakery protocol, from clauses $\delta_{2}$ and $\delta_{3}$, by using a generalization operator we introduce the new clause:

$$
\operatorname{gen}(A 2) \leftarrow A 2 \geq 1 \wedge B 2=0 \wedge \operatorname{sat}(\langle\text { wait, } A 2, \text { think, B2 }\rangle \text {, ef unsafe })
$$

Suitable generalization operators which ensure the termination of the UFV strategy for a large class of infinite state systems can be found in [9].

\subsubsection{Examples of Verification of Infinite State Systems}

In this section we use our verification method for proving various properties of infinite state systems. In particular, we consider the Bakery protocol [11] and we verify that it satisfies the mutual exclusion and starvation freedom properties. Then, at the end of the section, we report on some more experimental results concerning the proofs of properties of several other protocols.

Let us consider two agents $A$ and $B$ which want to access a shared resource in a mutual exclusive way by using the Bakery protocol. The state of agent $A$ is represented by a pair $\langle A 1, A 2\rangle$, where $A 1$ is an element of the set $\{$ think, wait, use $\}$ of control states, and $A 2$ is a counter that takes values from the set of natural numbers. Analogously, the state of agent $B$ is represented by a pair $\langle B 1, B 2\rangle$. The state of the system consisting of the two agents $A$ and $B$, whose states are $\langle A 1, A 2\rangle$ and $\langle B 1, B 2\rangle$, respectively, is represented by the 4 -tuple $\langle A 1, A 2, B 1, B 2\rangle$. The transition relation $t$ of the two agent system from an old state OldState to a new state NewState, is defined as follows:

TA. $t$ (OldState, NewState $) \leftarrow t A$ (OldState, NewState)

TB. $t$ (OldState, NewState $) \leftarrow t B$ (OldState, NewState $)$

where the transition relation $t A$ for the agent $A$ is given by the following clauses whose bodies are conjunctions of constraints (see also Figure 1.2):

A1. $t A(\langle$ think, $A 2, B 1, B 2\rangle,\langle$ wait $, A 21, B 1, B 2\rangle) \leftarrow A 21=B 2+1$

A2. $t A(\langle$ wait, $A 2, B 1, B 2\rangle,\langle$ use, $A 2, B 1, B 2\rangle) \leftarrow A 2<B 2$

A3. $t A(\langle$ wait, $A 2, B 1, B 2\rangle,\langle$ use, $A 2, B 1, B 2\rangle) \leftarrow B 2=0$

A4. $t A(\langle$ use, $A 2, B 1, B 2\rangle,\langle$ think, $A 21, B 1, B 2\rangle) \leftarrow A 21=0$

The following analogous clauses define the transition relation $t B$ for the agent $B$ : 
B1. $t B(\langle A 1, A 2$, think,$B 2\rangle,\langle A 1, A 2$, wait,$B 21\rangle) \leftarrow B 21=A 2+1$

B2. $t B(\langle A 1, A 2$, wait, $B 2\rangle,\langle A 1, A 2$, use,$B 2\rangle) \leftarrow B 2<A 2$

B3. $t B(\langle A 1, A 2$, wait, $B 2\rangle,\langle A 1, A 2$, use, $B 2\rangle) \leftarrow A 2=0$

B4. $t B(\langle A 1, A 2$, use, $B 2\rangle,\langle A 1, A 2$, think, $B 21\rangle) \leftarrow B 21=0$

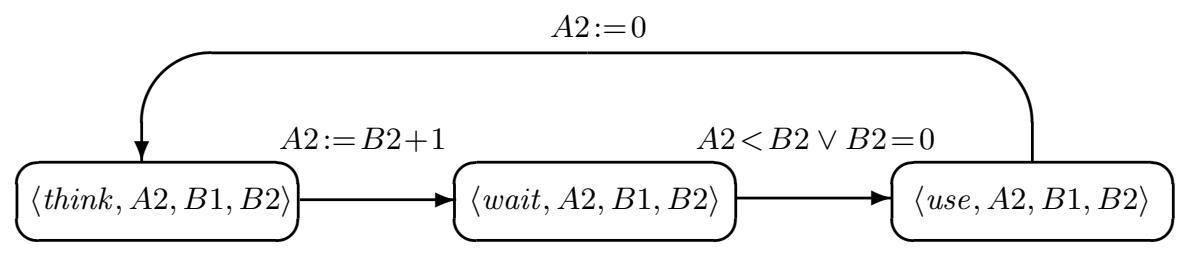

Figure 1.2. The Bakery protocol: a graphical representation of the transition relation $t A$ for the agent $A$. The assignment $X:=e$ on the arc from state $s_{1}$ to state $s_{2}$ tells us that the value of the variable $X$ in $s_{2}$ is obtained from the value of the expression $e$ in $s_{1}$. The boolean expression $b$ on the arc from state $s_{1}$ to state $s_{2}$ tells us that the transition from $s_{1}$ to $s_{2}$ takes place iff $b$ holds.

Notice that the two agent system has an infinite number of states, because counters may increase in an unbounded way, as indicated by the underlined states of the following computation path starting from the initial state $\langle$ think, 0 , think, 0$\rangle$ :

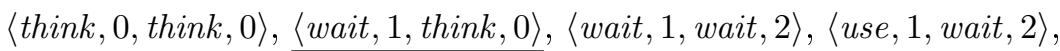

$\langle$ think, 0 , wait, 2$\rangle,\langle$ think, 0, use, 2$\rangle,\langle$ wait, 3 , use, 2$\rangle,\langle$ wait, 3 , think, 0$\rangle, \ldots$

We may apply our verification method for checking the mutual exclusion property of the Bakery protocol. This property is expressed by the CTL formula: $\neg$ ef unsafe, where for all states $s$,

elem ( $s$, unsafe) holds iff $s$ is of the form $\langle u s e, A 2$, use, $B 2\rangle$,

that is, unsafe holds iff both agents $A$ and $B$ are in the control state use.

The initial program $P_{\mathcal{K}}$ which encodes the Kripke structure of the Bakery protocol with two agents $A$ and $B$, is the following one:

1. $\operatorname{sat}(\langle$ use, $A 2$, use, B2 $\rangle$, unsafe $) \leftarrow$

2. $\operatorname{sat}(S, \neg F) \leftarrow \neg \operatorname{sat}(S, F)$

3. $\operatorname{sat}(S$, ef $F) \leftarrow \operatorname{sat}(S, F)$

4. $\operatorname{sat}(S$, ef $F) \leftarrow t(S, T) \wedge \operatorname{sat}(T$, ef $F)$

together with the clauses TA, TB, A1-A4, and B1-B4, which define the transition relation $t$. The initial clause $\delta_{0}$ for the mutual exclusion property is:

new $0_{m e} \leftarrow \operatorname{sat}(\langle$ think, 0 , think, 0$\rangle$, ᄀef unsafe $)$

For the Bakery protocol we may also want to prove the starvation freedom property which ensures that an agent, say $A$, which requests the shared resource, will eventually get it. This property is expressed by the CTL formula: $a g($ wait $A \rightarrow$ af use $A)$, which is equivalent to: $\neg$ ef $($ wait $A \wedge \neg$ af use $A)$. For the elementary properties wait $A$ and use $A$, the satisfaction relation is defined by the clauses: 


$$
\begin{aligned}
& \text { sat }(\langle\text { wait, } A 2, B 1, B 2\rangle, \text { wait } A) \leftarrow \\
& \text { sat }(\langle\text { use }, A 2, B 1, B 2\rangle, \text { use } A) \leftarrow
\end{aligned}
$$

For the starvation freedom property the initial clause $\delta_{0}$ is:

$$
\text { new } 0_{s f} \leftarrow \operatorname{sat}(\langle\text { think, } 0 \text {, think, } 0\rangle, \neg \text { ef }(\text { wait } A \wedge \neg \text { af use } A))
$$

The clauses for $\operatorname{sat}(X, \neg F)$, sat $(X, F 1 \wedge F 2)$, and $\operatorname{sat}(X$, ef $F)$ are clauses S2-S5 (see Section 1.2). We do not have the space here to list all clauses for $\operatorname{sat}(X$, af $F)$. These clauses include clause S6 (see Section 1.2) together with one or more clauses of the form S7 (see Section 1.2) for each state $s$ of the form $\langle A 1, A 2, B 1, B 2\rangle$, where $A 1$ and $B 1$ belong to $\{$ think, wait, use $\}$. For instance, the clause for the state $\langle$ think, $A 2$, think, $B 2\rangle$ is:

$$
\begin{aligned}
& \text { sat }(\langle\text { think, } A 2 \text {, think, B2 }\rangle \text {, af } F) \leftarrow A 21=B 2+1 \wedge B 21=A 2+1 \\
& \wedge \text { sat }(\langle\text { wait }, A 21, \text { think }, B 2\rangle, \text { af } F) \wedge \operatorname{sat}(\langle\text { think }, A 2, \text { wait, B21 }\rangle \text {, af } F)
\end{aligned}
$$

The remaining clauses for $\operatorname{sat}(X$, af $F)$ can be found in [9].

By using our experimental constraint logic program transformation system MAP [14] we have been able to automatically verify the mutual exclusion and the starvation freedom properties of the Bakery protocol.

We have verified some more properties of various other protocols by using our system MAP running on a Linux machine with a $900 \mathrm{MHz}$ clock, and the results of these experiments are reported in the following Table 1.1. The verification times we have obtained demonstrate that our system performs well w.r.t. the DMC system [6] and the other systems cited in $[6,7]$.

\begin{tabular}{l|l|c} 
Protocol & \multicolumn{1}{|c}{ Property } & Verification Time \\
\hline \hline Bakery & $\neg e f$ unsafe & 0.2 \\
(mutual exclusion) & ag $($ wait $A \rightarrow$ af use $A)$ & 2.3 \\
\hline Ticket & $\neg e f$ unsafe & 0.6 \\
(mutual exclusion) & $a g($ waitA $\rightarrow$ af useA $)$ & 3.0 \\
\hline Berkeley RISC & $\neg e f($ dirty $\geq 2)$ & 2.0 \\
(cache coherence) & $\neg e f($ dirty $\geq 1 \wedge$ shared $\geq 1)$ & 1.3 \\
\hline Xerox Dragon & $\neg e f($ dirty $\geq 2)$ & 1.3 \\
(cache coherence) & $\neg e f($ dirty $\geq 1 \wedge$ shared_clean $\geq 1)$ & 0.9 \\
& $\neg e f($ dirty $\geq 1 \wedge$ shared_dirty $\geq 1)$ & 1.0 \\
\hline DEC Firefly & $\neg e f($ dirty $\geq 2)$ & 0.4 \\
(cache coherence) & $\neg e f($ dirty $\geq 1 \wedge$ shared $\geq 1)$ & 0.4 \\
\hline Illinois University & $\neg e f($ dirty $\geq 2)$ & 0.3 \\
(cache coherence) & $\neg e f($ dirty $\geq 1 \wedge$ shared $\geq 1)$ & 0.3 \\
\hline MESI & $\neg e f($ dirty $\geq 2)$ & 0.3 \\
(cache coherence) & $\neg e f($ dirty $\geq 1 \wedge$ shared $\geq 1)$ & 0.2 \\
\hline
\end{tabular}

Table 1.1. Experimental results of the verification of some properties of various protocols. The protocols and the properties are taken from [6]. The verification time is expressed in seconds. 


\subsection{Conclusions and Related Work}

We have presented a method for verifying CTL properties of protocols for multiagent systems specified as constraint logic programs. For systems which have a finite number of states, the method is complete and can be used as a decision procedure. For systems which have an infinite number of states, the method may not terminate. However, for a large class of infinite state systems, the method terminates if we use suitable generalization operators. We have applied our method for proving safety and liveness properties of several infinite state protocols.

Our verification method is related to others presented in the literature for the proofs of properties of concurrent systems which use the logic programming paradigm. Among them we mention the following ones.

In [18] the authors present XMC, a model checking system implemented in the tabulation-based logic programming language XSB. XMC can verify temporal properties expressed in the alternation-free fragment of the $\mu$-calculus of finite state systems specified in a CCS-like language.

In [17] a model checker is presented for verifying CTL properties of finite state systems, by using logic programs with finite constraint domains that are closed under conjunction, disjunction, variable projection and negation. The verification process is performed by executing a constraint logic program encoding the semantics of CTL in an extended execution model that uses constructive negation and tabled resolution.

In [10] an automatic method for verifying safety properties of infinite state Petri nets with parametric initial markings is presented. The method constructs the reachability set of the Petri net being verified by computing the least fixpoint of a logic program with Presburger arithmetic constraints.

A method for the verification of some CTL properties of infinite state systems using constraint logic programming is described in [7]. Suitable constraint logic programs which encode the system and the property to be verified, are introduced, and then, the CTL properties are verified by computing exact and approximated least and greatest fixed points of those programs.

Finally, the use of program transformation for verifying properties of infinite state systems has been investigated in $[12,19]$. In particular, (i) specialization of logic programs and abstract interpretation are used in [12] for the verification of safety properties of infinite state systems, and (ii) unfold/fold transformation rules are applied in [19] for proving safety and liveness properties of parameterized finite state systems with various network topologies.

\section{Acknowledgements}

Many thanks to Dr. A. Jankowski, Prof. A. Skowron, and Dr. M. Szczuka for their kind invitation at the International Workshop MSRAS 2004. We would like also to thank Prof. G. Delzanno, Prof. S. Etalle, and Prof. M. Leuschel for helpful discussions and comments. 


\section{References}

1. K.R. Apt and R.N. Bol. Logic programming and negation: A survey. J. Logic Programming, 19, 20:9-71, 1994.

2. R.M. Burstall and J. Darlington. A transformation system for developing recursive programs. JACM, 24(1):44-67, January 1977.

3. L. Cardelli and A.D. Gordon. Mobile ambients. Theoretical Computer Science, 240(1):177-213, 2000.

4. W. Chen and D.S. Warren. Tabled evaluation with delaying for general logic programs. JACM, 43(1), 1996.

5. E.M. Clarke, O. Grumberg, and D. Peled. Model Checking. MIT Press, 2000.

6. G. Delzanno. Automatic verification of parametrized cache coherence protocol. In Proc. CAV 2000, LNCS 1855, 55-68. Springer, 2000.

7. G. Delzanno and A. Podelski. Model checking in CLP. In R. Cleaveland (ed.) Proc. TACAS '99, LNCS 1579, 223-239. Springer, 1999.

8. S. Etalle and M. Gabbrielli. Transformations of CLP modules. Theoretical Computer Science, 166:101-146, 1996.

9. F. Fioravanti, A. Pettorossi, and M. Proietti. Verifying CTL properties of infinite state systems by specializing constraint logic programs. R. 544, IASI-CNR, Roma, Italy, 2001.

10. L. Fribourg and H. Olsén. Proving safety properties of infinite state systems by compilation into Presburger arithmetic. In Proc. CONCUR '97, LNCS 1243, 96-107. Springer-Verlag, 1997.

11. L. Lamport. A new solution of Dijkstra's concurrent programming problem. CACM, 17(8):453-455, 1974.

12. M. Leuschel and T. Massart. Infinite state model checking by abstract interpretation and program specialization. In Proc. LOPSTR '99, LNCS 1817, 63-82. Springer, 1999.

13. J.W. Lloyd. Foundations of Logic Programming. Springer-Verlag, Berlin, 1987.

14. MAP group. The MAP transformation system. Available from: http://ww.iasi.rm.cnr.it/〜proietti/system.html, 1995-2004.

15. K. Marriott and P. Stuckey. Programming with Constraints: An Introduction. The MIT Press, 1998

16. R. Milner, J. Parrow, and D. Walker. A calculus of mobile processes. Part I and II. Information and Computation, 100(1):1-77, 1992.

17. U. Nilsson and J. Lübcke. Constraint logic programming for local and symbolic model-checking. In Proc. CL 2000, LNAI 1861, 384-398. Springer, 2000.

18. Y.S. Ramakrishna, C.R. Ramakrishnan, I.V. Ramakrishnan, S.A. Smolka, T. Swift, and D.S. Warren. Efficient model checking using tabled resolution. In Proc. $C A V$ '97, LNCS 1254, 143-154. Springer, 1997.

19. A. Roychoudhury, K. Narayan Kumar, C.R. Ramakrishnan, I.V. Ramakrishnan, and S.A. Smolka. Verification of parameterized systems using logic program transformations. In Proc. TACAS 2000, LNCS 1785, 172-187. Springer, 2000.

20. H. Seki. Unfold/fold transformation of stratified programs. Theoretical Computer Science, 86:107-139, 1991.

21. H. Tamaki and T. Sato. Unfold/fold transformation of logic programs. In S.A. Tärnlund (ed.) Proc. 2nd Int. Conf. Logic Programming, 127-138, Uppsala, Sweden, 1984. 